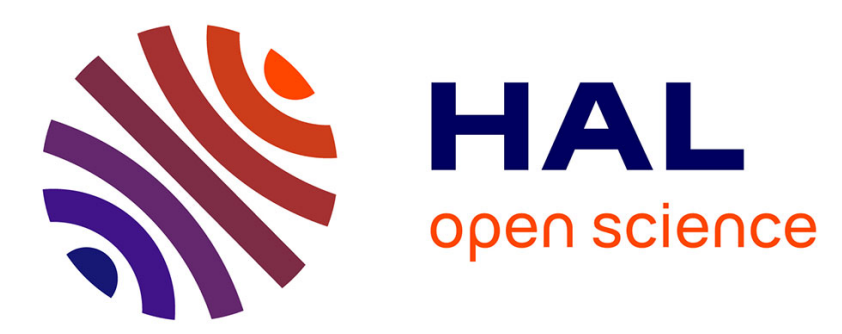

\title{
Calcul simultané de la polarisabilité et de l'énergie de transition électronique des molécules. Cas particulier de l'éthylène et du benzène
}

\author{
J. Barriol, Jérémi Regnier
}

\section{- To cite this version:}

J. Barriol, Jérémi Regnier. Calcul simultané de la polarisabilité et de l'énergie de transition électronique des molécules. Cas particulier de l'éthylène et du benzène. J. Phys. Radium, 1954, 15 (6), pp.528-530. 10.1051/jphysrad:01954001506052801 . jpa-00234985

\section{HAL Id: jpa-00234985 https://hal.science/jpa-00234985}

Submitted on 1 Jan 1954

HAL is a multi-disciplinary open access archive for the deposit and dissemination of scientific research documents, whether they are published or not. The documents may come from teaching and research institutions in France or abroad, or from public or private research centers.
L'archive ouverte pluridisciplinaire HAL, est destinée au dépôt et à la diffusion de documents scientifiques de niveau recherche, publiés ou non, émanant des établissements d'enseignement et de recherche français ou étrangers, des laboratoires publics ou privés. 


\section{GALGUL SIMULTANÉ DE LA POLARISABILITÉ ET DE L'ÉNERGIE DE TRANSITION ÉLEGTRONIQUE DES MOLÉGULES. GAS PARTIGULIER DE L ÉTHYLĖNE ET DU BENZĖNE}

Par J. BARRIOL et J. REGNIER, Faculté des Sciences de Nancy (France).

Dans une première partie, on traite en général le problème de la polarisabilité par la méthode variationnelle. On montre que $\alpha$ est peu sensible au choix de la fonction d'onde et que pour une forme simple de cette dernière, $\alpha$ et l'énergie de l'état perturbé sont obtenus exactement. Ces vues sont alors appliquées à $\mathrm{C}_{2} \mathrm{H}_{4}$ en prenant une fonction de Heitler-London corrigée. Dans une seconde partie, le cas de $\mathrm{C}_{2} \mathrm{H}_{4}$ est repris par la méthode $\mathrm{MO}$ simple, puis comparé à celui de $\mathrm{C}_{6} \mathrm{H}_{6}$ traité par les MO antisymétrisées. On donne aussi une méthode permettant d'évaluer la transition électronique moyenne dans $\mathrm{C}_{2} \mathrm{H}_{4}$.

\section{Première partie ( ${ }^{(1)}$.}

I. Rappelons tout d'abord brièvement les formules classiques de la théorie quantique de la polarisabilité

(1) La deuxième partie fera l'objet d'une publication ultérieure. d'un système atomique ou moléculaire à plusieurs électrons. A l'état normal; un tel système sera décrit par la connaissance de la fonction $\bigcup_{0}$, solution d'une certaine équation de Hamilton

$$
H_{0} \psi_{0}=E_{0} \psi_{0}
$$

Considérons la perturbation correspondant à l'établissement d'un champ électrique uniforme $f$, dirigé suivant l'axe $\mathrm{O} y$, par exemple. Le nouvel état possède l'énergie $E$ définie par le minimum de l'intégrale

$$
E=\int \psi^{\star}\left(H_{0}-e f y\right) \psi \mathrm{d} \tau=E_{0}-\frac{\mathrm{I}}{2} \alpha f^{2}
$$

( $y$ est mis en abrégé pour $\Sigma_{r} y_{r}$, correspondant aux divers électrons).

Le calcul de l'énergie $E$, et partant celui de la polarisabilité $\alpha$ s'effectue ainsi par la méthode variationnelle classique. Un tel calcul se poursuit habituellement en formant la fonction $\psi$ comme combi- 
naison linéaire de fonctions propres de l'opérateur $H_{0}$. Présentons rapidement ce calcul en se limitant à deux termes, ce qui n'altère en rien la généralité. La seule particularité que nous introduirons est de considérer que l'une des fonctions entrant dans cette combinaison, soit ici $\psi_{2}$, n'est pas nécessairement fonction propre de $H_{0}$. Nous prenons ainsi :

$$
\psi=\lambda \psi_{0}+\mu \psi_{1}+\nu \psi_{2}
$$

et posons que les trois fonctions $\psi_{0}, \psi_{1}, \psi_{2}$ sont normées et orthogonales entre elles deux à deux, choix toujours possible évidemment. La condition de normation et l'expression de l'énergie $E$ s'écrivent alors immédiatement

$$
\begin{gathered}
\lambda^{2}+\mu^{2}+\nu^{2}=\mathrm{I} \\
E=\lambda^{2} E_{0}+\mu^{2} E_{1}+\nu^{2} E_{2}-2 f \lambda \mu Y_{10}-2 f \lambda \nu Y_{20}
\end{gathered}
$$

en se limitant aux termes du second ordre et en posant

$$
\begin{gathered}
H_{0} ⿱_{1}=E_{1} \iota_{1}, \quad \int \psi_{2}^{\star} H_{0} \psi_{2} \mathrm{~d} \tau=E_{\Perp}, \\
e \int \psi_{1}^{\star} y \psi_{0} \mathrm{~d} \tau=Y_{10}, \quad e \int \psi_{\stackrel{2}{*}} y \psi_{0} \mathrm{~d} \tau=Y_{20} .
\end{gathered}
$$

La valeur de l'énergie correspondant au minimum de (2), compte tenu de (1), est racine de l'équation

$$
\left|\begin{array}{ccc}
E_{0}-E & -Y_{10} f & -Y_{20} f \\
-Y_{10} f & E_{1}-E & 0 \\
-Y_{20} f & 0 & E_{2}-E
\end{array}\right|=0 .
$$

Celle des racines de cette équation, qui nous intéresse ici, diffère de $E_{0}$ par le terme $-\frac{1}{2} \alpha f^{2}$, de sorte que l'on peut immédiatement écrire

$$
\dot{\alpha}=\frac{2 Y_{10}^{2}}{E_{1}-E_{0}}+\frac{2 Y_{20}^{2}}{E_{2}-E_{0}} .
$$

Cette formule classique se généralise sans difficulté au cas où l'on introduit un nombre quelconque de fonctions propres de $H_{0}$ dans l'expression de $\alpha$. La forme de cette expression suggère quelques remarques.

En premier lieu, l'adjonction de nouveaux termes dans l'expression de $\psi$ ne modifie en rien les termes déjà existant dans (3). Ces termes étant tous positifs, il en résulte que le calcul effectué avec un nombre insuffisant de termes doit fournir une valeur de $\alpha$ approchée par défaut.

En second lieu, la manière dont la polarisabilité $\alpha$ est liée à l'énergie $E$ implique que, de même que $E$, $\alpha$ est donné par une condition d'extremum, ce qui fait que la valeur calculée pour cette grandeur doit etre relativement peu sensible au choix de la fonction $\psi$.

Enfin remarquons que le calcul de chacun des termes de la somme (3) nécessite, d'une part la connaissance de l'intégrale $Y_{20}$ et, d'autre part, celle de l'énergie, en général beaucoup plus malaisée à obtenir.

C'est pourquoi il nous a paru intéressant d'essayer une méthode de calcul approximatif des polarisabilités correspondant à l'introduction d'une seule fonction $\Downarrow_{2}$ associée à $\psi_{0}$ pour constituer $\psi$. Les calculs effectués sur les molécules diatomiques nous ont conduit à prendre

$$
\varphi_{2}=\frac{y \psi_{0}}{K_{r}}
$$

en introduisant la constante de normation $K_{3}$. Une telle fonction présente d'ailleurs l'intérêt de permettre un calcul particulièrement simple de l'énergie, en utilisant la relation suivante qui se démontre immédiatement :

$$
H_{0} \sum_{r} y_{r}=\sum_{r} y_{r} H_{0}-\frac{h^{2}}{4 \pi^{2} m} \sum_{r} \frac{\partial}{\partial y_{r}} .
$$

Il en résulte alors l'expression suivante pour l'énergie moyenne $E_{2}$ dans l'état $\psi_{2}$ :

$$
\begin{aligned}
K_{y}^{2} E_{:}= & \int \Sigma y_{r} \psi_{0} H_{0} \Sigma y_{r} \psi_{0} \mathrm{~d} z=\int\left(\Sigma y_{r}\right)^{2} \psi_{0} H_{0} \psi_{0} \mathrm{~d} z \\
& -\frac{h^{2}}{4 \pi^{2} m} \sum_{r, s} \int y_{r} \frac{\partial \psi_{0}}{\partial y_{s}} \mathrm{~d} \tau .
\end{aligned}
$$

La première intégrale du membre de droite se simplifie, compte tenu du fait que $\psi_{0}$ est fonction propre de $H_{0}$. Pour ce qui est du second membre, il se transforme aisément en faisant apparaître une intégrale de surface qui est égale à zéro. Il vient donc, en définitive, sans faire d'approximation :

$$
E_{2}-E_{0}=\frac{\nu h^{2}}{8 \pi^{2} m K_{1}^{2}}
$$

pour un système comportant $v$ électrons. Par ailleurs, on a également

$$
K_{y}^{2} \int \psi_{2}^{2} \mathrm{~d} \tau=K_{y}^{2}=\int y^{2} \psi_{0}^{2} \mathrm{~d} \tau,
$$

d'où la valeur de la polarisabilité, donnée par la formule (3) :

$$
\alpha=\frac{\mathrm{I} 6 \pi^{2} m e^{2} K_{y}^{2}}{\nu h^{2}}=\frac{4 K_{Y}^{4}}{\nu a_{0}}
$$

en introduisant le rayon $a_{0}=\frac{h^{2}}{4 \pi^{2} m e^{2}}$ de l'orbite $1 s$ de l'hydrogène.

Nous nous proposons de discuter l'emploi de cette formule approchée dans un certain nombre de cas particuliers.

II. Incrément de réfraction moléculaire de la double liaison éthylénique. - Nous admettons que cet incrément est dû aux seuls électrons $\pi$. Le champ électrique est appliqué suivant $O y$ et les orbitales $2 p_{z}$ de Slater, centrées sur les carbones $\mathrm{A}$ et $\mathrm{B}$, seront désignées par $a$ et $b, \mathrm{O} z$ étant perpendiculaire au plan de la molécule. Les deux électrons sont numérotés 1 et 2 et $R$ est la distance carbone-carbone.

Nous postulons de plus que, dans l'état fondamental, la distribution des électrons $\pi$ est représentée par la fonction de Craig [1] :

$$
\begin{aligned}
\psi_{0}=N\{a(\mathrm{I}) b(2)+a(2) b(\mathrm{I}) \\
+\lambda[a(\mathrm{I}) a(2)+b(\mathrm{I}) b(2)]\},
\end{aligned}
$$


où

$$
N-2=2\left[(\mathrm{I}+\lambda S)^{2}+(\lambda+S)^{2}\right]
$$

exprime la condition de normation.

Calculons en premier lieu la polarisabilité suivant $\mathrm{O} y$. La valeur de $K_{y}$ introduite par (4), s'obtient par la formule

$$
K_{r}^{2}=\int\left(y_{1}+y_{2}\right)^{2} \psi_{0}^{2} \mathrm{~d} \tau_{1} \mathrm{~d} \tau_{2} .
$$
type

Nous négligeons dans ce calcul les intégrales du

$$
\int y^{2} a b \mathrm{~d} \tau
$$

ce qui se justifie par le fait que $y$ est nul lorsque le produit $a b$ est maximum. On obtient ainsi

$$
K_{r}^{2}=\frac{2 C_{y}^{2}+\lambda^{2}\left(R^{2}+2 C_{y}^{2}\right)+\lambda S\left(R^{2}+4 C_{7}^{2}\right)}{(S+\lambda)^{2}+(I+\lambda S)^{2}},
$$

avec

$$
C_{\gamma}^{2}=\int y_{a}^{2} a^{2} \mathrm{~d} \tau,
$$

l'indice $a$ de $y_{a}$ rappelant que l'origine est maintenant prise sur le carbone $A$.

La formule (5) fournit alors la valeur de la polarisabilité

$$
\alpha_{y}=\frac{2}{a_{0}}\left[\frac{2 C_{y}^{2}+\lambda^{2}\left(R^{2}+2 C_{y}^{2}\right)+\lambda S\left(R^{2}+4 C_{y}^{2}\right)}{-(S+\lambda)^{2}+(\mathrm{I}+\lambda S)^{2}}\right]^{2} .
$$

Pour la polarisabilité suivant $\mathrm{O} x$, le calcul se poursuit de la même manière en introduisant

$$
K_{x}^{2}=\frac{2 C_{x}^{2}\left(\mathrm{I}+\lambda^{2}+2 \lambda S\right)}{(S+\lambda)^{2}+(\mathrm{I}+\lambda S)^{2}} .
$$

Le calcul est un peu plus compliqué en ce qui concerne l'axe $\mathrm{O} z$, du fait que l'on ne peut négliger a priori l'intégrale

$$
\int z^{2} a b \mathrm{~d} \tau
$$

Nous prendrons cette intégrale égale à

$$
\zeta^{2} \int a b \mathrm{~d} \tau=\zeta^{2} S
$$

en désignant par $\zeta$ la cote pour laquelle le produit $a b$ est maximum, $y$ étant nul. Il vient alors

$$
K_{z}^{2}=2 \frac{\left(C_{z}^{2}+\zeta^{2} S^{2}\right)\left(\mathrm{I}+\lambda^{2}\right)+2 \lambda S\left(C_{z}^{2}+\zeta^{2}\right)}{(S+\lambda)^{2}+(\mathrm{I}+\lambda S)^{2}} .
$$

Passons maintenant au calcul de la polarisabilité moyenne définie par

$$
\bar{\alpha}=\frac{\alpha_{x}+\alpha_{y}+\alpha_{z}}{3} .
$$

Nous obtenons

$$
\bar{\alpha}=\frac{2}{3 a_{0}}\left(K_{x}^{4}+K_{r}^{4}+K_{z}^{4}\right) .
$$

Nous avons évalué les quantités $\mathrm{C}_{x}, C_{y}$ et $C_{z}$ en utilisant l'approximation de Slater dans la représentation des orbitales atomiques. Désignons par $Z$ la charge effective des noyaux de carbone, c'està-dire corrigée de l'effet écran des autres électrons. Il vient

$$
\begin{gathered}
C_{x}^{2}=C_{y}^{2}=6\left(\frac{a_{0}}{Z}\right)^{2}, \quad C_{z}^{2}=18\left(\frac{a_{0}}{Z}\right)^{2}, \\
\zeta^{2}=2\left(\frac{a_{0}}{Z}\right)^{2}+\left(\frac{a_{0}}{Z}\right) R .
\end{gathered}
$$

Avec les valeurs [1] :

$$
R=\mathrm{I}, 34 \AA \mathbf{A}, \quad Z=3,43, \quad \lambda=0,264,
$$

on obtient pour incrément de double liaison éthylénique

$$
P=2,8 \mathbf{I}
$$

en bon accord avec la valeur expérimentale 2,94, si l'on se souvient que la valeur calculée pour $\alpha$ est certainement approchée par défaut. D'ailleurs la valeur expérimentale 2,94 correspond à la réfractivité mesurée pour la raie $D$ du sodium. L'accord serait meilleur si l'on comparait le résultat trouvé à une réfractivité extrapolée pour une longueur d'onde infinie. Il est à remarquer, d'autre part, que l'on n'a pas tenu compte de la variation de la réfractivité de la liaison $\mathrm{C}-\mathrm{C}$ tant en ce qui concerne la distance internucléaire que le type d'hybridation.

On notera enfin que la polarisabilité varie comme $\frac{\mathbf{I}}{Z^{4}}$, d'où l'influence considérable de la charge d'écran. La distance carbone-carbone joue un rôle moins important.

[1] Craig D. P. - Proc. Roy. Soc., A, 1949-1950, 200, 272-283. 